\title{
EXISTENCE RESULTS ON THE ONE-DIMENSIONAL DIRICHLET PROBLEM SUGGESTED BY THE PIECEWISE LINEAR CASE
}

\author{
M. ARIAS ${ }^{1}$
}

\begin{abstract}
We study the existence of solutions of a two-point boundary value problem at resonance in which the nonlinearity grows at most linearly. Sharp results for the linear growth of the nonlinearity in each direction are obtained.
\end{abstract}

1. Introduction. We study the existence of solutions in the sense of Carathéodory of the nonlinear boundary value problem at resonance

$$
u^{\prime \prime}+n^{2} u+g(u)=f(x), \quad u(0)=u(\pi)=0,
$$

where $g: R \rightarrow R$ is a continuous function which may grow linearly, $f \in L^{2}(0, \pi)$ and $n$ is an integer number.

One of the first results related to this problem, but in the nonresonant case, was due to Loud [8] (see also [7]).

When $n=1$ (resonance at the first eigenvalue), (1.1) has been studied by many authors (see, for instance, $[\mathbf{1}, \mathbf{3}$ and $\mathbf{9}]$ ), but few (see [4]) have studied problem (1.1) for $n \geq 2$ (resonance at an eigenvalue greater than the first one).

Considering the linear problem, i.e., $g(u)=\gamma u$ in (1.1), it is known that (1.1) is solvable for each $f \in L^{2}(0, \pi)$ provided that $0<\gamma<(n+1)^{2}-n^{2}$. Then, one can hope that if the nonlinearity $g$ behaves like $\gamma u$ with $0<\gamma<(n+1)^{2}-n^{2}$, the result holds in the nonlinear case.

In this line we obtain, as a consequence of our main theorem, the following result:

THEOREM 1. Assume that

(a) There exist $\gamma, C \in R, 0<\gamma<(n+1)^{2}-n^{2}$, such that

$$
|g(u)| \leq \gamma|u|+C, \quad u \in R .
$$

(b) For $n>1$ there exist $s>0$ and $L \in R$ such that

$$
g(u) \leq g(v)+L, \quad \text { if } v-u \geq s .
$$

(c)

$$
\begin{aligned}
\bar{g}(-\infty) \int_{0}^{\pi} \sin ^{+} n x d x-\underline{g}(+\infty) & \int_{0}^{\pi} \sin ^{-} n x d x<\int_{0}^{\pi} f(x) \sin n x d x \\
& <\underline{g}(+\infty) \int_{0}^{\pi} \sin ^{+} n x d x-\bar{g}(-\infty) \int_{0}^{\pi} \sin ^{-} n x d x .
\end{aligned}
$$

Received by the editors June 17, 1985.

1980 Mathematics Subject Classification. Primary 34B15, 34C15, 34C25.

Key words and phrases. Resonance, a priori bounds, Sturm comparison theory, Dirichlet piecewise linear problem.

${ }^{1}$ The author wishes to acknowledge support from CAICYT, Ministerio de Educacion y Ciencia, Spain. 
Then (1.1) has at least one solution.

(Here, $\bar{g}(-\infty)=\limsup _{u \rightarrow-\infty} g(u), \underline{g}(+\infty)=\liminf _{u \rightarrow+\infty} g(u), v^{+}(x)=$ $\max \{v(x), 0\}$ and $\left.v^{-}(x)=\max \{-v(x), 0\}, v \in C[0, \pi]\right)$.

Condition (a) in this theorem is sharp. Actually, when $g(u)=\left[(n+1)^{2}-n^{2}\right] u$, (1.1) becomes $u^{\prime \prime}+(n+1)^{2} u=f, u(0)=u(\pi)=0$, which has a solution if and only if $\int_{0}^{\pi} f(x) \sin (n+1) x d x=0$. However, we know that if $g(u)=\mu^{2} u^{+}-\nu^{2} u^{-}$ (piecewise linear problem), problem (1.1) is solvable for each $f \in L^{2}(0, \pi)$ provided that $\mu$ and $\nu$ satisfy a certain relation which allows $\mu$ to be very large if $\nu$ is sufficiently small, and vice versa (see $\S 2$ ). So, if instead of using the linear problem as a model, we use this piecewise linear problem, we obtain

THEOREM 2 (MAIN THEOREM). Suppose that conditions (b) and (c) of Theorem 1 hold and

( $\left.\mathrm{a}^{\prime}\right)$ There exist $\mu, \nu \geq n, \alpha, \beta, k, K \in R$ such that

$$
\begin{gathered}
\alpha \leq g(u) \leq\left(\mu^{2}-n^{2}\right)|u|+K, \quad u \geq 0, \\
\beta \geq g(u) \geq-\left(\nu^{2}-n^{2}\right)|u|+k, \quad u \leq 0,
\end{gathered}
$$

with

(i) $n / 2 \mu+(n+2) / 2 \nu>1,(n+2) / 2 \mu+n / 2 \nu>1$ if $n$ is even,

(ii) $(n+1) / 2 \mu+(n+1) / 2 \nu>1$ if $n$ is odd.

Then (1.1) has at least one solution.

Note that if $\mu=\nu=\gamma$, condition ( $\mathrm{a}^{\prime}$ ) is condition (a) of Theorem 1 .

Condition $\left(\mathrm{a}^{\prime}\right)$ is also sharp, as we will show in the final remarks and, in contrast to condition (a), allows $g$ to have a very large linear growth in the positive direction provided that it has sufficiently small growth in the negative one, and vice versa.

Our proof is based on establishing a priori bounds for possible solutions of (1.1) and takes some ideas from [1], where Ahmad, by comparison with the linear problem, proves Theorem 1 in the case $n=1$.

The author wishes to thank Professor R. Kannan for suggesting the problem to her and Professors R. Ortega and P. Martínez Amores for their valuable comments and suggestions about this work.

2. The piecewise linear case. Consider the piecewise linear problem

$$
u^{\prime \prime}+\mu^{2} u^{+}-\nu^{2} u^{-}=f, \quad u(0)=u(\pi)=0 .
$$

This problem has been extensively studied by Dancer $[\mathbf{4}]$. There he proves that (2.1) has a nontrivial solution for $f=0$ if and only if

$$
\begin{aligned}
&(\mu, \nu) \in A_{0}=\bigcup_{k=1}^{\infty}\left[\left\{(\mu, \nu) \in R^{2}: \frac{k}{\mu}+\frac{k}{\nu}=1\right\}\right. \\
& \cup\left\{(\mu, \nu) \in R^{2}: \frac{k-1}{\mu}+\frac{k}{\nu}=1\right\} \\
&\left.\cup\left\{(\mu, \nu) \in R^{2}: \frac{k}{\mu}+\frac{k-1}{\nu}=1\right\}\right]
\end{aligned}
$$

Moreover, he proves that if $(\mu, \nu) \in A_{1}$, where $A_{1}$ is the shaded set in Figure 1, (2.1) has a solution for an arbitrary $f \in L^{2}(0, \pi)$, whereas if $(\mu, \nu) \in R^{2}-\bar{A}_{1}$ there exists $f \in C^{\infty}[0, \pi]$ such that $(2.1)$ has no solution. 


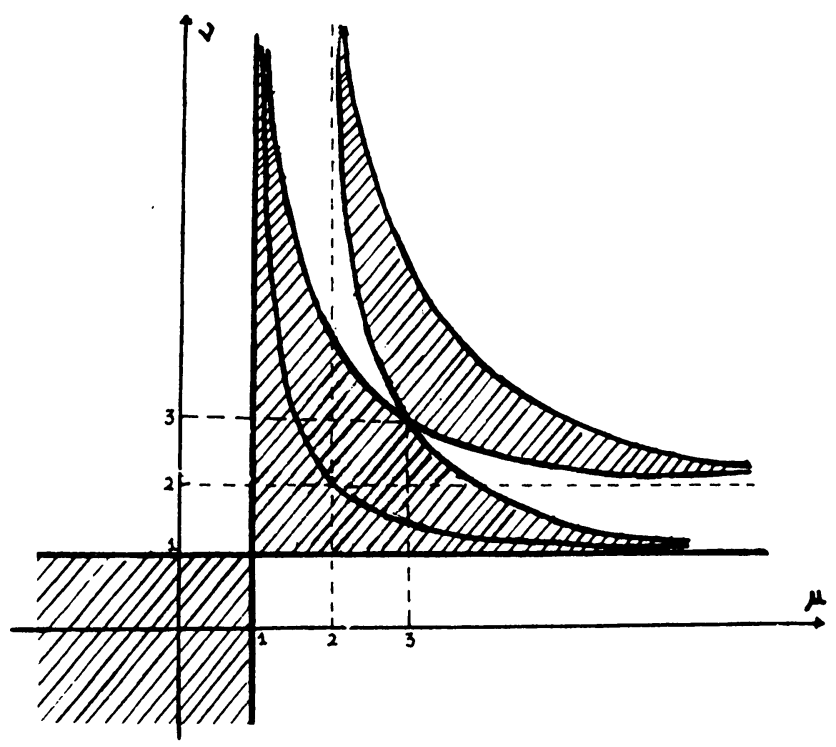

FIGURE 1

These results suggest the following

LEMMA 1. Let $\tilde{\mu}, \tilde{\nu} \in L^{2}(0, \pi)$. Suppose there exist nonnegative constants $\mu, \nu$ satisfying (i) and (ii) of hypothesis ( $\left.\mathrm{a}^{\prime}\right)$ of Theorem 2 and such that $n^{2} \leq \tilde{\mu}(x) \leq \mu^{2}$, a.e. $(0, \pi), n^{2} \leq \tilde{\nu}(x) \leq \nu^{2}$, a.e. $(0, \pi)$. Then the problem

$$
\begin{gathered}
u^{\prime \prime}+\tilde{\mu}(x) u^{+}-\tilde{\nu}(x) u^{-}=0 \quad \text { a.e. }(0, \pi), \\
u(0)=u(\pi)=0,
\end{gathered}
$$

has a nontrivial solution if and only if

$$
\begin{aligned}
& (\sigma \sin n x)^{+} \tilde{\mu}(x)=(\sigma \sin n x)^{+} n^{2} \quad \text { a.e. }(0, \pi), \\
& (\sigma \sin n x)^{-} \tilde{\nu}(x)=(\sigma \sin n x)^{-} n^{2} \quad \text { a.e. }(0, \pi),
\end{aligned}
$$

where $\sigma= \pm 1$. In this case if $\phi$ is a nontrivial solution of $(2.2), \phi(x)=C \sigma \sin n x$, with $C>0$.

Proof. Suppose (2.3) is not verified and $n$ is even. (The proof when $n$ is odd is similar.)

Let $\phi$ be a nontrivial solution of $(2.2)$. Then either $\phi^{\prime}(0)>0$ or $\phi^{\prime}(0)<0$. Suppose, for instance, $\phi^{\prime}(0)>0$. The other case is analogous. By the Sturm comparison theorem (S.C.T), there exists $t_{1} \in[\pi / \mu, \pi / n]$ such that $\phi^{\prime \prime}+\tilde{\mu}(x) \phi=0$, a.e. $\left(0, t_{1}\right), \phi(0)=\phi\left(t_{1}\right)=0$, and $\phi^{\prime}\left(t_{1}\right)<0$. Again by S.T.C. there exists $t_{2} \in$ $[\pi / \mu+\pi / \nu, 2 \pi / n]$ such that $\phi^{\prime \prime}+\tilde{\nu}(x) \phi=0$, a.e. $\left(t_{1}, t_{2}\right), \phi\left(t_{1}\right)=\phi\left(t_{2}\right)=0$.

In this way we found that there exists $t_{n} \in[n \pi / 2 \mu+n \pi / 2 \nu, \pi]$ such that $\phi^{\prime \prime}+$ $\tilde{\nu}(x) \phi=0$, a.e. $\left(t_{n-1}, t_{n}\right), \phi\left(t_{n-1}\right)=\phi\left(t_{n}\right)=0$. But, since (2.3) is not true, there exists a subset of $(0, \pi)$ with positive measure in which $\tilde{\mu}(x) \sin ^{+} n x>n^{2} \sin ^{+} n x$ or $\tilde{\nu}(x) \sin ^{-} n x>n^{2} \sin ^{-} n x$. Therefore, by S.C.T., $t_{n}<\pi$. Then, since $\phi(\pi)=0$, there exists $t_{n+1} \leq \pi$ such that $\phi^{\prime \prime}+\tilde{\mu}(x) \phi=0$, a.e. $\left(t_{n}, t_{n+1}\right), \phi\left(t_{n}\right)=\phi\left(t_{n+1}\right)=0$, 
but this is impossible because, by S.C.T.,

$$
t_{n+1} \geq \frac{\pi}{\mu}+t_{n} \geq \frac{(n+2) \pi}{2 \mu}+\frac{n \pi}{2 \nu}>\pi
$$

On the other hand, if (2.3) is verified, $\phi(x)=C \sigma \sin n x, C>0$, is a nontrivial solution of (2.2). By a similar argument we can prove that these are the only solutions of (2.2) when (2.3) holds.

\section{Proof of Theorem 2. We need}

LEMMA 2. Let $g: R \rightarrow R$ be a continuous function satisfying hypothesis (b) of Theorem 1 (for $n=1$, suppose there exist $\alpha, \beta \in R$ such that $g(u) \geq \alpha, u \geq 0$ and $g(u) \leq \beta, u \leq 0)$, and let $\left\{u_{i}\right\}_{i \in N}$ be a sequence in $C^{1}[0, \pi]$ such that $\left\|u_{i}\right\| \rightarrow_{i \rightarrow \infty} \infty$ and $u_{i} /\left\|u_{i}\right\| \rightarrow \sigma \sin (n \cdot), \sigma= \pm 1$, in $C^{1}[0, \pi]$ (i.e., $u_{i}(x) /\left\|u_{i}\right\| \rightarrow \sigma \sin n x$ and $u_{i}^{\prime}(x) /\left\|u_{i}\right\| \rightarrow n \sigma \cos n x$ uniformly in $\left.[0, \pi]\right)$. Then

$$
\begin{aligned}
& \liminf _{i \rightarrow \infty} \sigma \int_{0}^{\pi} g\left(u_{i}(x)\right) \sin n x d x \\
& \quad \geq \underline{g}(+\infty) \int_{0}^{\pi}(\sigma \sin n)^{+} x d x-\bar{g}(-\infty) \int_{0}^{\pi}(\sigma \sin n)^{-} x d x .
\end{aligned}
$$

(Here, $\left.\|u\|=\max _{x \in[0, \pi]}|u(x)|.\right)$

PROOF. For simplicity we only consider the cases $n=1$ and $n=2$. Suppose $\sigma=1$. If $\sigma=-1$ the proof is similar. If $n=1$, then for each $\varepsilon>0$ there exists $i_{\varepsilon} \in N$ such that $g\left(u_{i}(x)\right) \geq \underline{g}(+\infty)-\varepsilon, x \in[\varepsilon, \pi-\varepsilon]$ and $u_{i}(x)>0, x \in(0, \pi)$, if $i \geq i_{\varepsilon}$. Thus, for $i \geq i_{\varepsilon}$,

$$
\begin{aligned}
\int_{0}^{\pi} g\left(u_{i}(x)\right) \sin x d x \geq & \alpha \int_{0}^{\varepsilon} \sin x d x+\underline{g}(+\infty) \int_{\varepsilon}^{\pi-\varepsilon} \sin x d x \\
& -\varepsilon \int_{\varepsilon}^{\pi-\varepsilon} \sin x d x+\alpha \int_{\pi-\varepsilon}^{\pi} \sin x d x .
\end{aligned}
$$

Letting $\varepsilon \rightarrow 0$, we obtain (3.1) in this case.

Now suppose $n=2$. For each $\varepsilon>0$ there exists $i_{\varepsilon} \in N$ such that $g\left(u_{i}(x)\right)>$ $\underline{g}(+\infty)-\varepsilon, x \in[\varepsilon, \pi / 2-\varepsilon], u_{i}(x)>0, x \in(0, \pi / 2-\varepsilon), g\left(u_{i}(x)\right)<\bar{g}(-\infty)+\varepsilon$, $x \in[\pi / 2+\varepsilon, \pi-\varepsilon], u_{i}(x)<0, x \in(\pi / 2+\varepsilon, \pi)$ and there exists a unique point $x_{i} \in(\pi / 2-\varepsilon, \pi / 2+\varepsilon)$ such that $u_{i}\left(x_{i}\right)=0$, if $i \geq i_{\varepsilon}$. We can suppose, taking a subsequence if necessary, that $x_{i} \geq \pi / 2, i \geq i_{\varepsilon}$, or $x_{i} \leq \pi / 2, i \geq i_{\varepsilon}$. Consider the first case (the other is analogous).

For each $\delta>0$ there exist $0<\varepsilon(\delta)<\delta$ and $i_{\delta} \in N$ such that $\left\|u_{i}(x)\right\| \leq \delta\left|u_{i}\right|$, $x \in(\pi / 2-\varepsilon, \pi / 2+\varepsilon)$ and $u_{i}(x)>(1-\delta)\left\|u_{i}\right\|, x \in(\pi / 4-\varepsilon, \pi / 4+\varepsilon)$, if $i \geq i_{\delta}$.

Let $m_{i}=\inf \left\{g(u): u>(1-\delta)\left\|u_{i}\right\|\right\}$ and $M_{i}=\sup \left\{g(u): 0<u<\delta\left\|u_{i}\right\|\right\}$. Thus, for $i \geq \max \left\{i_{\delta}, i_{\varepsilon(\delta)}\right\}$, since $g(u) \geq g(-s)-L, u \geq 0$ and $g(u) \leq g(s)+L, u \leq 0$, 
we have

$$
\begin{aligned}
\int_{0}^{\pi} g\left(u_{i}(x)\right) & \sin 2 x d x \geq(g(-s)-L) \int_{0}^{\varepsilon} \sin 2 x d x \\
& +(\underline{g}(+\infty)-\varepsilon) \int_{\varepsilon}^{\pi / 4-\varepsilon} \sin 2 x d x+m_{i} \int_{\pi / 4-\varepsilon}^{\pi / 4+\varepsilon} \sin 2 x d x \\
& +(\underline{g}(+\infty)-\varepsilon) \int_{\pi / 4+\varepsilon}^{\pi / 2-\varepsilon} \sin 2 x d x+(g(-s)-L) \int_{\pi / 2-\varepsilon}^{\pi / 2} \sin 2 x d x \\
& +M_{i} \int_{\pi / 2}^{x_{i}} \sin 2 x d x+(g(s)+L) \int_{x_{i}}^{\pi / 2+\varepsilon} \sin 2 x d x \\
& +(\bar{g}(-\infty)+\varepsilon) \int_{\pi / 2+\varepsilon}^{\pi-\varepsilon} \sin 2 x d x+(g(s)+L) \int_{\pi-\varepsilon}^{\pi} \sin 2 x d x .
\end{aligned}
$$

From hypothesis (b), $M_{i} \leq m_{i}+L$ if $\delta<\frac{1}{2}$ and $i$ sufficiently large. Then letting $\delta \rightarrow 0$ (so $\varepsilon \rightarrow 0$ ), we obtain (3.1).

To prove Theorem 2 we use a well-known continuation theorem.

Consider the family of Dirichlet problems

$$
\begin{gathered}
u^{\prime \prime}+n^{2} u+\lambda g(u)=\lambda f \quad \text { a.e. }(0, \pi), \\
u(0)=u(\pi)=0,
\end{gathered}
$$

with $\lambda \in(0,1)$. It is enough (see $[6$, p. 40 and 2]) to prove that

(1) There exists $R_{0}>0$ such that

$$
\left[\int_{0}^{\pi}(g(R)-f(x)) \sin n x d x\right]\left[\int_{0}^{\pi}(g(-R)-f(x)) \sin n x d x\right]<0
$$

for each $R>R_{0}$.

(2) There exists $C>0$ such that $\|u\| \leq C$ for all solutions $(\lambda, u)$ of (3.2).

Condition (1) follows immediately from hypotheses (a) and (c). We prove (2). Suppose (2) is false. Then for each $i \in N$ there exist solutions $\left(\lambda_{i}, u_{i}\right)$ of (3.2) such that $\left\|u_{i}\right\| \geq i$. Define $v_{i}=u_{i} /\left\|u_{i}\right\| ;\left\|v_{i}\right\|=1$ for all $i \in N$ and $v_{i}$ verifies

$$
\begin{gathered}
v_{i}^{\prime \prime}+n^{2} v_{i}+\frac{\lambda_{i}}{\left\|u_{i}\right\|} g\left(u_{i}\right)=\frac{\lambda_{i}}{\left\|u_{i}\right\|} f \quad \text { a.e. }(0, \pi), \\
v_{i}(0)=v_{i}(\pi)=0 .
\end{gathered}
$$

From hypothesis $\left(\mathrm{a}^{\prime}\right)$ we have $|g(u)| \leq r|u|+\tau$, where $r=\max \left(\mu^{2}-n^{2}, \nu^{2}-n^{2}\right)$ and $\tau=\max (|\alpha|,|\beta|,|k|,|K|)$. Then $\left\{g\left(u_{i}\right) /\left\|u_{i}\right\|\right\}$ is uniformly bounded in $L^{2}(0, \pi)$, and, since $v_{i}$ is a solution of $(3.3),\left\{v_{i}^{\prime \prime}\right\}$ is uniformly bounded in $L^{2}(0, \pi)$. Thus $\left\{v_{i}^{\prime}\right\}$ and, therefore, $\left\{v_{i}\right\}$ are uniformly bounded and equicontinuous. Hence, there exists a subsequence, relabeled $\left\{v_{i}\right\}$, and $v \in C^{1}[0, \pi]$ such that $v_{i} \rightarrow v$ in $C^{1}[0, \pi]$.

We claim that there exist $\tilde{\mu}, \tilde{\nu} \in L^{2}(0, \pi)$, with $n^{2} \leq \tilde{\mu}(x) \leq \mu^{2}, n^{2} \leq \tilde{\nu}(x) \leq \nu^{2}$ a.e. such that $v$ satisfies

$$
\begin{gathered}
v^{\prime \prime}+\tilde{\mu}(x) v^{+}-\tilde{\nu}(x) v^{-}=0 \quad \text { a.e. }(0, \pi), \\
v(0)=v(\pi)=0 .
\end{gathered}
$$

Indeed, suppose that $v(x)>0, x \in(a, b), 0 \leq a<b \leq \pi$, and let $I_{j}=\left(a_{j}, b_{j}\right) \subset$ $(a, b), a_{j}<b_{j}, a_{j} \downarrow a, b_{j} \uparrow b$, be a sequence of intervals such that $\bigcup_{j=1}^{\infty} I_{j}=(a, b)$. 
Let $j \in N$ be fixed. Since $v(x)>0$ in $\bar{I}_{j}, v_{i}(x)>0$ in $\bar{I}_{j}$ for $i$ sufficiently large, and so $u_{i}(x) \rightarrow+\infty$ uniformly in $\bar{I}_{j}$. We can suppose $u_{i}(x)>0, x \in \bar{I}_{j}$. From $\left(\mathrm{a}^{\prime}\right)$,

$$
-\left|\frac{\alpha}{u_{i}(x)}\right| \leq \frac{\lambda_{i} g\left(u_{i}(x)\right)}{u_{i}(x)} \leq \mu^{2}-n^{2}+\left|\frac{K}{u_{i}(x)}\right|, \quad x \in I_{j} .
$$

Thus, $\left\{\lambda_{i} g\left(u_{i}\right) / u_{i}\right\}$ is uniformly bounded in $L^{2}\left(I_{j}\right)$, and there exists $\mu_{j} \in L^{2}\left(I_{j}\right)$ such that $\lambda_{i} g\left(u_{i}\right) / u_{i} \rightarrow c \rightarrow \infty c \rightarrow \infty \mu_{j}$ in $L^{2}\left(I_{j}\right)$. Moreover, from (3.5) and Mazur's Theorem, $0 \leq \mu_{j} \leq \mu^{2}-n^{2}$ a.e. $I_{j}$.

Integrating (3.3) between $a_{j}$ and $x$ in $I_{j}$ and letting $i \rightarrow \infty$, we obtain

$$
v^{\prime}(x)-v^{\prime}\left(a_{j}\right)+\int_{a_{j}}^{x}\left(n^{2}+\mu_{j}(t)\right) v(t) d t=0 .
$$

Therefore, $v^{\prime}$ is absolutely continuous in $I_{j}$ and $v$ satisfies $v^{\prime \prime}+\left(n^{2}+\mu_{j}(x)\right) v=0$ a.e. $I_{j}$.

Evidently, if $k \neq j, \mu_{j}(x)=\mu_{k}(x)$ a.e. $x \in I_{j} \cap I_{k}$. Define $\tilde{\mu} \in L^{2}(a, b)$ by $\tilde{\mu}(x)=n^{2}+\mu_{j}(x)$; if $x \in I_{j}$, then $n^{2} \leq \tilde{\mu}(x) \leq \mu^{2}$ a.e. $(a, b)$ and $v$ satisfies $v^{\prime \prime}+\tilde{\mu}(x) v^{+}=0$ a.e. $(a, b)$.

Analogously, if $v(x)<0, x \in(a, b)$, then there exists $\tilde{\nu} \in L^{2}(a, b), n^{2} \leq \tilde{\nu}(x) \leq$ $\nu^{2}$ a.e. $(a, b)$ such that $v^{\prime \prime}-\tilde{\nu}(x) v^{-}=0$ a.e. $(a, b)$. This proves our claim.

By Lemma 1 , since $\|v\|=1$, it follows that $v(x)=\sigma \sin n x, \sigma= \pm 1$, and then, by Lemma 2, we have (3.1). But

$$
\int_{0}^{\pi} g\left(u_{i}(x)\right) \sin n x d x=\int_{0}^{\pi} f(x) \sin n x d x \quad \text { for all } i \in N
$$

because $\left(\lambda_{i}, u_{i}\right)$ is a solution of $(3.2)$ for each $i \in N$; thus

$$
\sigma \int_{0}^{\pi} f(x) \sin n x d x \geq \underline{g}(+\infty) \int_{0}^{\pi}(\sigma \sin n)^{+} x d x-\bar{g}(-\infty) \int_{0}^{\pi}(\sigma \sin n)^{-} x d x
$$

which contradicts hypothesis (c). So, condition (2) is verified, and the theorem is proved.

4. Final remarks. (1) Hypothesis (b) is satisfied if $g$ is monotone nondecreasing or bounded. For $n=1$ this hypothesis is not needed. We do not know if it is possible to prove the theorem without hypothesis (b) in all cases. This condition was used by Ward [10] in another context.

(2) We can prove the theorem by substituting "there exist $r, d>0$ such that $|g(s, u)| \geq r|g(u)|-d$, if $|u| \geq d$ and $s \geq d$ " for hypothesis (b).

This is Property $P$ in [4], so our theorem generalizes some of the results in [5] where it is assumed that $\lim _{u \rightarrow \pm \infty} g(u) / u$ exists.

(3) As we said in $\S 1$, condition $\left(a^{\prime}\right)$ in the theorem is sharp. Indeed, taking $g(u)=\left(\mu^{2}-n^{2}\right) u^{+}-\left(\nu^{2}-n^{2}\right) u^{-}$with $\mu, \nu>n$ and $\mu$ and $\nu$ satisfying some equality in (i) or (ii), Dancer [5] proved that there exists $f \in L^{2}(0, \pi)$ for which (1.1) has no solution.

(4) An analogous theorem can be proved when we substitute "there exist $\mu, \nu<$ $n, \alpha, \beta, k, K \in R$ such that $\alpha \geq g(u) \geq\left(\mu^{2}-n^{2}\right)|u|+K, u \geq 0, \beta \leq g(u) \leq$ $-\left(\nu^{2}-n^{2}\right)|u|+k, u \leq 0$, with

(i') $n / 2 \mu+(n-2) / 2 \nu<1,(n-2) / 2 \mu+n / 2 \nu<1$ if $n$ is even,

(ii') $(n-1) / 2 \mu+(n-1) / 2 \nu<1$ if $n$ is odd $(\mu, \nu<1$, for $n=1)$ "

for hypothesis $\left(a^{\prime}\right)$, and we consider (b) and (c) with the reversed inequalities. 


\section{REFERENCES}

1. S. Ahmad, A resonance problem in which the nonlinearity may grow linearly, Proc. Amer. Math. Soc. 92 (1984), 381-384.

2. L. Cesari, Functional analysis, nonlinear differential equations and the alternative method, Nonlinear Functional Analysis and Differential Equations (L. Cesari, R. Kannan and J. D. Schuur, eds.), Dekker, New York, 1977, pp. 1-197.

3. L. Cesari and R. Kannan, Existence of solutions of a nonlinear differential equation, Proc. Amer. Math. Soc. 88 (1983), 605-613.

4. E. N. Dancer, On the Dirichlet problem for weakly nonlinear elliptic partial differential equations, Proc. Roy. Soc. Edinburgh Sect. A 76 (1977), 283-300.

5. _ Boundary-value problems for weakly nonlinear ordinary differential equations, Bull. Austral. Math. Soc. 15 (1976), 321-328.

6. R. W. Gaines and J. L. Mawhin, Coincidence degree and nonlinear differential equations, Lecture Notes in Math., vol. 568, Springer-Verlag, Berlin and New York, 1977.

7. D. E. Leach, On Poincaré's perturbation theorem and a theorem of W. S. Loud, J. Differential Equations 7 (1970), 34-53.

8. W. S. Loud, Periodic solutions of nonlinear differential equations of Duffing type, Proc. U.S. Japan Seminar on Differential and Functional Equations, Benjamin, New York, 1967, pp. 199-224.

9. J. L. Mawhin, J. R. Ward and M. Willen, Necessary and sufficient conditions for the solvability of nonlinear two-point boundary value problem, preprint.

10. J. R. Ward, Perturbations with some superlinear growth for a class of second order elliptic boundary value problems, Nonlinear Anal. 6 (1982), 307-374.

Departamento de Ecuaciones Funcionales, FaCultad de Ciencias, UniversiDAD DE GRANADA, 18071 GRANADA, SPAIN 